\title{
ON THE EXISTENCE OF POSITIVE SOLUTIONS OF ORDINARY DIFFERENTIAL EQUATIONS
}

\author{
L. H. ERBE AND HAIYAN WANG
}

(Communicated by Hal L. Smith)

\begin{abstract}
We study the existence of positive solutions of the equation $u^{\prime \prime}+$ $a(t) f(u)=0$ with linear boundary conditions. We show the existence of at least one positive solution if $f$ is either superlinear or sublinear by a simple application of a Fixed Point Theorem in cones.
\end{abstract}

\section{INTRODUCTION}

In this paper we shall consider the second-order boundary value problem (BVP)

$$
\begin{gathered}
u^{\prime \prime}+a(t) f(u)=0, \quad 0<t<1 ; \\
\alpha u(0)-\beta u^{\prime}(0)=0, \\
\gamma u(1)+\delta u^{\prime}(1)=0 .
\end{gathered}
$$

The following conditions will be assumed throughout:

(A.1) $f \in C([0, \infty),[0, \infty))$,

(A.2) $a \in C([0,1],[0, \infty))$ and $a(t) \not \equiv 0$ on any subinterval of $[0,1]$.

(A.3) $\alpha, \beta, \gamma, \delta \geq 0$ and $\rho:=\gamma \beta+\alpha \gamma+\alpha \delta>0$.

The BVP (1.1), (1.2) arises in many different areas of applied mathematics and physics; see $[1-3,6,12,13]$ for some references along this line. Additional existence results may be found in $[4,7,8,10,11]$. Our purpose here is to give an existence result for positive solutions to the BVP (1.1), (1.2), assuming that $f$ is either superlinear or sublinear. We do not require any monotonicity assumptions on $f$. To be precise, we introduce the notation

$$
f_{0}:=\lim _{u \rightarrow 0} \frac{f(u)}{u}, \quad f_{\infty}:=\lim _{u \rightarrow \infty} \frac{f(u)}{u} .
$$

Thus, $f_{0}=0$ and $f_{\infty}=\infty$ correspond to the superlinear case, and $f_{0}=\infty$ and $f_{\infty}=0$ correspond to the sublinear case. By a positive solution of (1.1), (1.2) we understand a solution $u(t)$ which is positive on $0<t<1$ and satisfies

Received by the editors June 8, 1992.

1991 Mathematics Subject Classification. Primary 34B15.

Key words and phrases. Boundary value problems, positive solution, superlinear and sublinear, fixed point theorem in cones. 
the differential equation (1.1) for $0<t<1$ and the boundary conditions (1.2). By a change of variable, the existence of a positive solution of (1.1), (1.2) may be shown to be equivalent to the existence of a positive radial solution of the semilinear elliptic equation $\Delta u+g(|x|) f(u)=0$ in the annulus $R_{1}<|x|<R_{2}$ subject to certain boundary conditions for $|x|=R_{1}$ and $|x|=R_{2}$. (Here $|x|$ denotes the Euclidean norm.) We refer to [11] for some additional details.

\section{EXISTENCE RESULTS}

The main result of this paper is

Theorem 1. Assume (A.1)-(A.3) hold. Then the BVP (1.1), (1.2) has at least one positive solution in the case

(i) $f_{0}=0$ and $f_{\infty}=\infty$ (superlinear), or

(ii) $f_{0}=\infty$ and $f_{\infty}=0$ (sublinear).

It will be seen in the proof that Theorem 1 is also valid for the more general equation

$$
u^{\prime \prime}+f(t, u)=0
$$

with the same boundary conditions (1.2), provided we assume a certain uniformity with respect to the $t$ variable. We state this more general result as

Corollary 1. Assume $f$ is continuous, $f(t, u) \geq 0$ for $t \in[0,1]$, and $u \geq 0$ with $f(t, u) \not \equiv 0$ on any subinterval of $[0,1]$ for $u>0$; and let condition (A.3) hold. Then the BVP $(1.1)^{*},(1.2)$ has at least one positive solution in the case

(i) $\lim _{u \rightarrow 0+} \max _{t \in[0,1]} \frac{f(t, u)}{u}=0$ and $\lim _{u \rightarrow \infty} \min _{t \in[0,1]} \frac{f(t, u)}{u}=\infty$, or

(ii) ${ }^{*} \quad \lim _{u \rightarrow 0+} \min _{t \in[0,1]} \frac{f(t, u)}{u}=\infty$ and $\lim _{u \rightarrow \infty} \max _{t \in[0,1]} \frac{f(t, u)}{u}=0$.

The proof of Theorem 1 will be based on an application of the following Fixed Point Theorem due to Krasnoselskii [9]. The proof of Corollary 1 follows from the proof of Theorem 1 with obvious slight modifications which we shall omit.

Theorem $2[4,9]$. Let $E$ be a Banach space, and let $K \subset E$ be a cone in $E$. Assume $\Omega_{1}, \Omega_{2}$ are open subsets of $E$ with $0 \in \Omega_{1}, \bar{\Omega}_{1} \subset \Omega_{2}$, and let

$$
A: K \cap\left(\bar{\Omega}_{2} \backslash \Omega_{1}\right) \rightarrow K
$$

be a completely continuous operator such that either

(i) $\|A u\| \leq\|u\|, u \in K \cap \partial \Omega_{1}$, and $\|A u\| \geq\|u\|, u \in K \cap \partial \Omega_{2}$; or

(ii) $\|A u\| \geq\|u\|, u \in K \cap \partial \Omega_{1}$, and $\|A u\| \leq\|u\|, u \in K \cap \partial \Omega_{2}$.

Then $A$ has a fixed point in $K \cap\left(\bar{\Omega}_{2} \backslash \Omega_{1}\right)$.

We will apply the first and second parts of the above Fixed Point Theorem to the superlinear and sublinear cases, respectively.

Proof of Theorem 1. Superlinear case. Suppose then that $f_{0}=0$ and $f_{\infty}=\infty$. We wish to show the existence of a positive solution of (1.1), (1.2). Now (1.1), (1.2) has a solution $u=u(t)$ if and only if $u$ solves the operator equation

$$
u(t)=\int_{0}^{1} k(t, s) a(s) f(u(s)) d s:=A u(t), \quad u \in C[0,1] .
$$


Here $k(t, s)$ denotes the Green's function for the BVP

$$
\begin{gathered}
u^{\prime \prime}=0 ; \\
\alpha u(0)-\beta u^{\prime}(0)=0, \\
\gamma u(1)+\delta u^{\prime}(1)=0
\end{gathered}
$$

and is explicitly given by

$$
k(t, s)= \begin{cases}\frac{1}{\rho}(\gamma+\delta-\gamma t)(\beta+\alpha s), & 0 \leq s \leq t \leq 1, \\ \frac{1}{\rho}(\beta+\alpha t)(\gamma+\delta-\gamma s), & 0 \leq t \leq s \leq 1 .\end{cases}
$$

We let $K$ be the cone in $C[0,1]$ given by

$$
K=\left\{u \in C[0,1]: u(t) \geq 0, \min _{1 / 4 \leq t \leq 3 / 4} u(t) \geq M\|u\|\right\}
$$

where $\|u\|=\sup _{[0,1]}|u(t)|$ and

$$
M=\min \left\{\frac{\gamma+4 \delta}{4(\gamma+\delta)}, \frac{\alpha+4 \beta}{4(\alpha+\beta)}\right\} .
$$

We define

$$
\varphi(t):=(\gamma+\delta-\gamma t), \quad \psi(t):=\beta+\alpha t, \quad 0 \leq t \leq 1,
$$

so that

$$
k(t, s)= \begin{cases}\frac{1}{\rho} \varphi(t) \psi(s), & 0 \leq s \leq t \leq 1, \\ \frac{1}{\rho} \varphi(s) \psi(t), & 0 \leq t \leq s \leq 1 .\end{cases}
$$

Observe that $k(t, s) \leq \frac{1}{\rho} \varphi(s) \psi(s)=k(s, s), 0 \leq t, s \leq 1$, so that, if $u \in K$, then

$$
A u(t)=\int_{0}^{1} k(t, s) a(s) f(u(s)) d s \leq \int_{0}^{1} k(s, s) a(s) f(u(s)) d s
$$

and hence

$$
\|A u\| \leq \int_{0}^{1} k(s, s) a(s) f(u(s)) d s .
$$

Furthermore, for $\frac{1}{4} \leq t \leq \frac{3}{4}$

so

$$
\frac{k(t, s)}{k(s, s)}=\left\{\begin{array} { l l } 
{ \frac { \varphi ( t ) } { \varphi ( s ) } , } & { s \leq t , } \\
{ \frac { \psi ( t ) } { \psi ( s ) } , } & { t \leq s ; }
\end{array} \quad \left\{\begin{array}{ll}
\frac{\gamma+4 \delta}{4(\gamma+\delta)}, & s \leq t, \\
\frac{\alpha+4 \beta}{4(\alpha+\beta)}, & t \leq s,
\end{array}\right.\right.
$$

Hence, if $u \in K$,

$$
\frac{k(t, s)}{k(s, s)} \geq M, \quad \frac{1}{4} \leq t \leq \frac{3}{4} .
$$

$$
\begin{aligned}
\min _{1 / 4 \leq t \leq 3 / 4} A u(t) & =\min _{1 / 4 \leq t \leq 3 / 4} \int_{0}^{1} k(t, s) a(s) f(u(s)) d s \\
& \geq M \int_{0}^{1} k(s, s) a(s) f(u(s)) d s \geq M\|A u\| .
\end{aligned}
$$


Therefore, $A K \subset K$. Moreover, it is easy to see that $A: K \rightarrow K$ is completely continuous.

Now, since $f_{0}=0$, we may choose $H_{1}>0$ so that $f(u) \leq \eta u$, for $0<u \leq$ $H_{1}$, where $\eta>0$ satisfies

$$
\eta \int_{0}^{1} k(s, s) a(s) d s \leq 1
$$

Thus, if $u \in K$ and $\|u\|=H_{1}$, then from (2.7) and (2.9)

$$
A u(t) \leq \int_{0}^{1} k(s, s) a(s) f(u(s)) \leq\|u\|, \quad 0 \leq t \leq 1 .
$$

Now if we let

$$
\Omega_{1}:=\left\{u \in E:\|u\|<H_{1}\right\}
$$

then (2.10) shows that

$$
\|A u\| \leq\|u\|, \quad u \in K \cap \partial \Omega_{1} .
$$

Further, since $f_{\infty}=\infty$, there exists $\widehat{H}_{2}>0$ such that $f(u) \geq \mu u, u \geq \widehat{H}_{2}$, where $\mu>0$ is chosen so that

$$
M \mu \int_{1 / 4}^{3 / 4} k\left(\frac{1}{2}, s\right) a(s) d s \geq 1 .
$$

Let $H_{2}:=\max \left\{2 H_{1}, \widehat{H}_{2} / M\right\}$ and $\Omega_{2}:=\left\{u \in E:\|u\|<H_{2}\right\}$. Then $u \in K$ and $\|u\|=H_{2}$ implies

$$
\min _{1 / 4 \leq t \leq 3 / 4} u(t) \geq M\|u\| \geq \widehat{H}_{2}
$$

and so

$$
\begin{aligned}
A u\left(\frac{1}{2}\right) & =\int_{0}^{1} k\left(\frac{1}{2}, s\right) a(s) f(u(s)) d s \geq \int_{1 / 4}^{3 / 4} k\left(\frac{1}{2}, s\right) a(s) f(u(s)) d s \\
& \geq \mu \int_{1 / 4}^{3 / 4} k\left(\frac{1}{2}, s\right) a(s) u(s) d s \geq \mu M\|u\| \int_{1 / 4}^{3 / 4} k\left(\frac{1}{2}, s\right) a(s) d s \geq\|u\| .
\end{aligned}
$$

Hence, $\|A u\| \geq\|u\|$ for $u \in K \cap \partial \Omega_{2}$.

Therefore, by the first part of the Fixed Point Theorem, it follows that $A$ has a fixed point in $K \cap \bar{\Omega}_{2} \backslash \Omega_{1}$ such that $H_{1} \leq\|u\| \leq H_{2}$. Further, since $k(t, s)>0$, it follows that $u(t)>0$ for $0<t<1$. This completes the superlinear part of the theorem.

Sublinear case. Suppose next that $f_{0}=\infty$ and $f_{\infty}=0$. We first choose $H_{1}>0$ such that $f(u) \geq \hat{\eta} u$ for $0<u \leq H_{1}$, where

$$
\hat{\eta} M \int_{1 / 4}^{3 / 4} k\left(\frac{1}{2}, s\right) a(s) d s \geq 1
$$

( $M$ is as in the first part of the proof). Then for $u \in K$ and $\|u\|=H_{1}$ we have

$$
\begin{aligned}
A u\left(\frac{1}{2}\right) & =\int_{0}^{1} k\left(\frac{1}{2}, s\right) a(s) f(u(s)) d s \\
& \geq \int_{1 / 4}^{3 / 4} k\left(\frac{1}{2}, s\right) a(s) f(u(s)) d s \geq \hat{\eta} \int_{1 / 4}^{3 / 4} k\left(\frac{1}{2}, s\right) a(s) u(s) d s \\
& \geq \hat{\eta} M\|u\| \int_{1 / 4}^{3 / 4} k\left(\frac{1}{2}, s\right) a(s) d s \geq\|u\| \quad[\text { by }(2.14)] .
\end{aligned}
$$


Thus, we may let $\Omega_{1}:=\left\{u \in E:\|u\|<H_{1}\right\}$ so that

$$
\|A u\| \geq\|u\| \text { for } u \in K \cap \partial \Omega_{1} .
$$

Now, since $f_{\infty}=0$, there exists $\widehat{H}_{2}>0$ so that $f(u) \leq \lambda u$ for $u \geq \widehat{H}_{2}$ where $\lambda>0$ satisfies

$$
\lambda \int_{0}^{1} k(s, s) a(s) d s \leq 1
$$

We consider two cases:

Case (i). Suppose $f$ is bounded, say $f(u) \leq N$ for all $u \in(0, \infty)$. In this case choose $H_{2}:=\max \left\{2 H_{1}, N \int_{0}^{1} k(s, s) a(s) d s\right\}$ so that for $u \in K$ with $\|u\|=H_{2}$ we have

$$
A u(t)=\int_{0}^{1} k(t, s) a(s) f(u(s)) d s \leq N \int_{0}^{1} k(s, s) a(s) d s \leq H_{2}
$$

and therefore $\|A u\| \leq\|u\|$.

Case (ii). If $f$ is unbounded, then let $H_{2}>\max \left\{2 H_{1}, \hat{H}_{2}\right\}$ and such that

$$
f(u) \leq f\left(H_{2}\right) \text { for } 0<u \leq H_{2} \text {. }
$$

(We are able to do this since $f$ is unbounded.)

Then for $u \in K$ and $\|u\|=H_{2}$ we have

$$
\begin{aligned}
A u(t) & =\int_{0}^{1} k(t, s) a(s) f(u(s)) d s \leq \int_{0}^{1} k(s, s) a(s) f(u(s)) d s \\
& \leq \int_{0}^{1} k(s, s) a(s) f\left(H_{2}\right) d s \leq \lambda H_{2} \int_{0}^{1} k(s, s) a(s) d s \leq H_{2}=\|u\|
\end{aligned}
$$

Therefore, in either case we may put

$$
\Omega_{2}:=\left\{u \in E:\|u\|<H_{2}\right\},
$$

and for $u \in K \cap \partial \Omega_{2}$ we have $\|A u\| \leq\|u\|$. By the second part of the Fixed Point Theorem it follows that BVP (1.1), (1.2) has a positive solution, and this completes the proof of the theorem.

\section{REFERENCES}

1. C. Bandle, C. V. Coffman, and M. Marcus, Nonlinear elliptic problems in annular domains, J. Differential Equations 69 (1987), 322-345.

2. C. Bandle and M. K. Kwong, Semilinear elliptic problems in annular domains, J. Appl. Math. Phys. 40 (1989), 245-257.

3. C. V. Coffman and M. Marcus, Existence and uniqueness results for semilinear Dirichlet problems in Annuli, Arch. Rational Mech. Anal. 108 (1989), 293-307.

4. K. Deimling, Nonlinear functional analysis, Springer, New York, 1985.

5. L. H. Erbe, Boundary value problems for ordinary differential equations, Rocky Mountain J. Math. 1 (1970), 709-729.

6. X. Garaizar, Existence of positive radial solutions for semilinear elliptic problems in the annulus, J. Differential Equations 70 (1987), 69-72.

7. G. B. Gustafson and K. Schmitt, Nonzero solutions of boundary value problems for second order ordinary and delay-differential equations, J. Differential Equations 12 (1972), 127147. 
8. Georges Iffland, Positive solutions of a problem of Emden-Fowler type with a free boundary, SIAM J. Math. Anal. 18 (1987), 283-292.

9. M. A. Krasnoselskii, Positive solutions of operator equations, Noordhoff, Groningen, 1964.

10. R. A. Moore and Z. Nehari, Nonoscillation theorems for a class of nonlinear differential equations, Trans. Amer. Math. Soc. 93 (1959), 30-52.

11. J. Santanilla, Nonnegative solutions to boundary value problems for nonlinear first and second order ordinary differential equations, J. Math. Anal. Appl. 126 (1987), 397-408.

12. Haiyan Wang, On the existence of positive solutions for semilinear elliptic equations in the annulus, J. Differential Equations (to appear).

13. J. S. W. Wong, On the generalized Emden-Fowler equation, SIAM Rev. 17 (1975), 339-360.

Department of Mathematics, University of Alberta, Edmonton, Alberta, Canada T6G 2G1

Department of Mathematics, Michigan State University, East lansing, Michigan, 48824 